Aus dem Institut

für Geschichte der Medizin an der Universität Münster (Westfalen)

\title{
Jean Riolan jun. (1580-1657) \\ im Streit mit Paul Marquart Schlegel (1605-1653) um die Blutbewegungslehre Harveys
}

\author{
Ein Beitrag zur Geschichte und Psychologie \\ des wissenschaftlichen Irrtums \\ Von K.E. Rотнsснuн, Münster (Westfalen)

\section{Einleitung und Fragestellung}

Wie die Weltgeschichte, so kennt auch die Wissenschaftsgeschichte Sieger und Besiegte. Den ersteren flicht sie Lorbeerkränze, den Unterlegenen bleibt der Spott der Nachwelt. So ging es auch mit William Harvey und dem Pariser Anatomen Jean Riolan. Sie sind im Schwarz-Weiß-Kontrast ${ }^{1}$ in die Geschichte eingegangen. Denn nur ein störrischer Esel konnte, so meint man, so hartnäckig den Blutkreislauf leugnen. Ole Worm ${ }^{2}$ schreibt an Thomas Bartholinus am 16. Juni 1654 über Riolan: «Er gilt zwar als gebildet und ist wegen seiner anatomischen Arbeiten geschätzt. Bis heute aber konnten weder Sitten, noch Musen, noch höfischer Dienst, noch der Zahn der Zeit ihn weich machen. Man muß annehmen, er habe seine Kinderstube bei alten Vetteln gehabt, denen die Streitsucht ein Vergnügen bereitet, die sich in zänkischen Ausfällen überbieten und den guten Ruf anderer in den Dreck zu ziehen versuchen.» ${ }^{3}$ Nun war weder Riolan alleine gehässig, noch lag alle Wahrheit bei seinen Gegnern. Das zeigt u.a. eine nähere Betrachtung der beiden Streitschriften, die in der Sache der Kreislauflehre zwischen Paul Marquart Schlegel (1605-1653) in Hamburg und Jean Riolan (fils) (1580-1657) in Paris 1650 bzw. 1652 gewechselt worden sind. Darin zeigt sich Schlegel nicht viel weniger aggressiv als Rio-

1 Man vergleiche z.B. F. Garrison, An Introduction to the History of Medicine, 4. ed., Philadelphia 1929, S. 248, "pedantic Riolan", "tympanitic Philistine".

2 Ole Worm (1588-1654), Schüler von Plater, Bauhin, Fabricius und Riolan sen., später Professor der Medizinischen Fakultät in Kopenhagen.

${ }^{3}$ Zit. nach B. J. GotTLIEB, Die Gelehrtenrepublik der Harvey-Zeit. Zeugnisse und Briefe an und um William Harvey (1642-1656), Ann. Univ. Saraviensis. Med. 8 (1960) 13-36. 


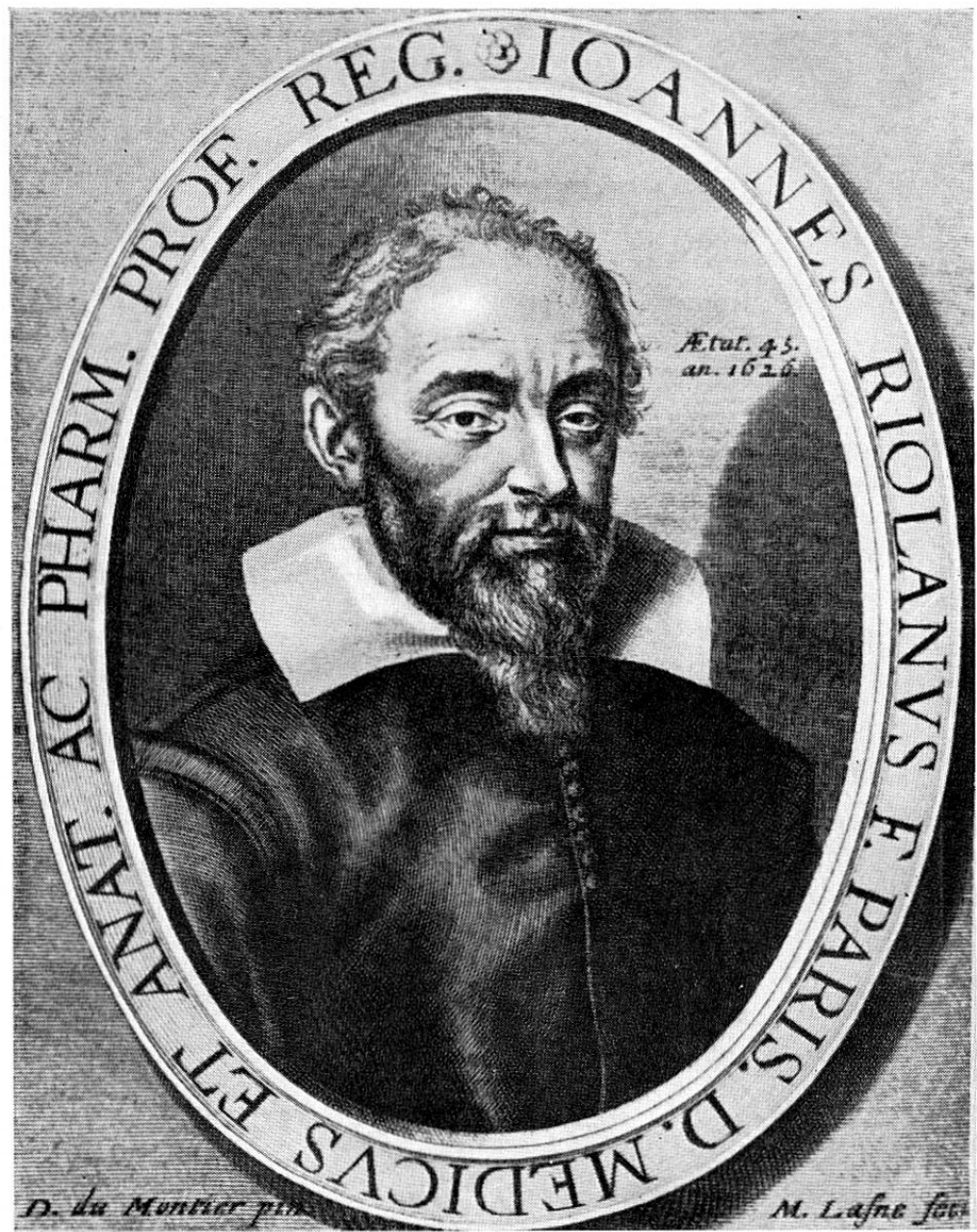

Abb. 3. Porträt des Jean Riolan jun. (1580-1657).

Stich aus dem Jahre 1626 von Michel Lasne

(aus R. N. Wegner, Das Anatomenbildnis ...) 


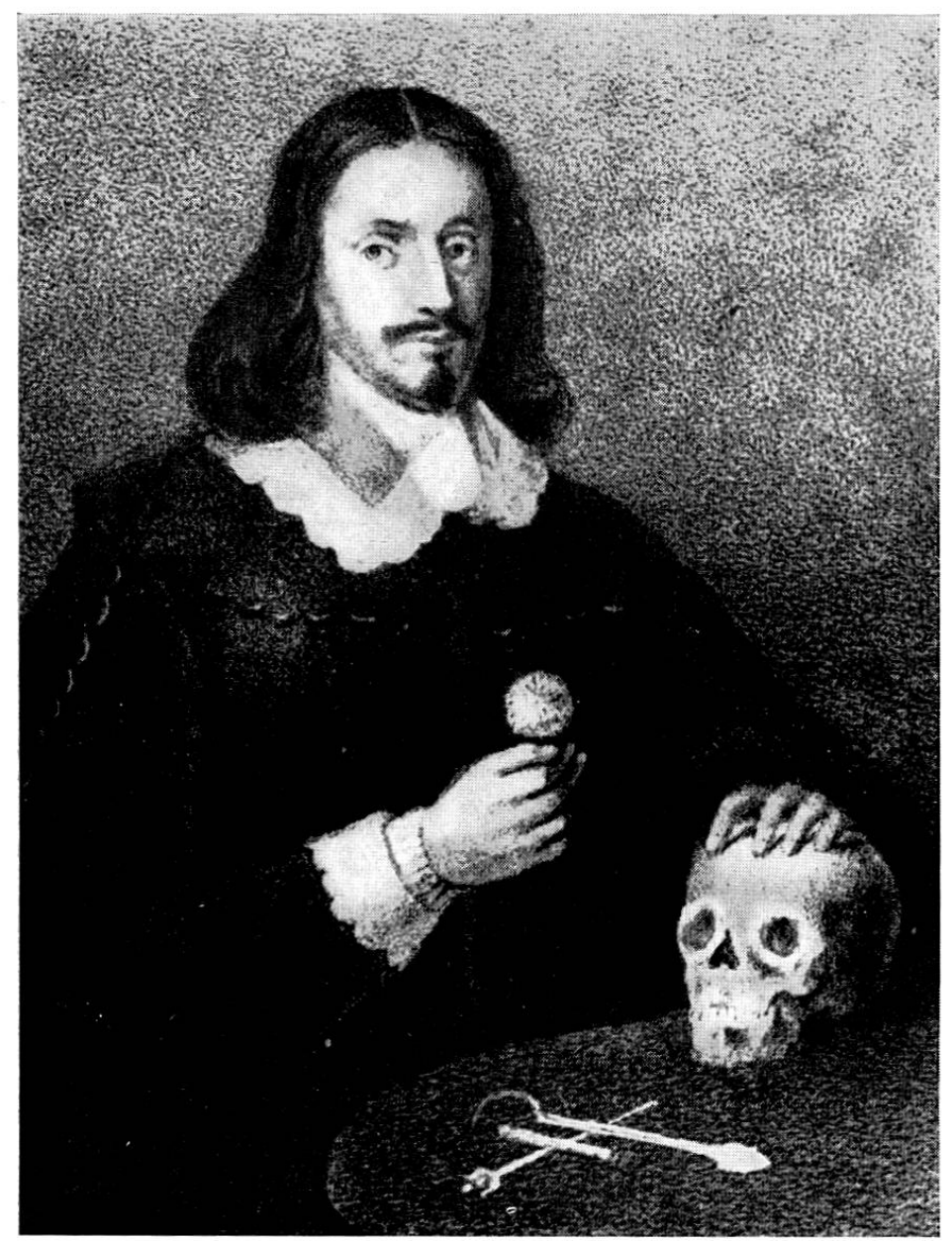

Abb. 4. Porträt des Paul Marquart Schlegel (1605-1653). Gemälde eines unbekannten Künstlers, im Besitz der Staatsund Universitätsbibliothek Hamburg 
lan, und, rein sachlich gesehen, sind Schlegels Argumente weniger originell als diejenigen von Riolan. Harveys Lehre war zu jener Zeit eben doch nur erst eine vorzügliche Theorie; sie ermangelte noch des letzten Beweisstückes ${ }^{4}$ und war mit gewissen ärztlichen Erfahrungen und Gepflogenheiten der damaligen Zeit schwer in Einklang zu bringen. Das hat Riolan viel schärfer als viele andere gesehen und ausgesprochen. Wie sehr sogar noch Harveys Anhänger mit Verständnisschwierigkeiten kämpften, zeigt z.B. Thomas Bartholinus (1616-1680). Er gibt noch in seiner Anatomia refor$m_{a t a^{5}}$ von 1651 an, daß der feinere Teil des Blutes durch das Septum cordis in die linke.Kammer gelangt, der gröbere geht zur Reinigung durch die Lungen. Er glaubt auch nach wie vor, daß die Rußteilchen des linken Herzens über die Vena arteriosa und die Lunge ins Freie ausgeschieden werden. Riolan war sicher der bedeutendste Leugner des Kreislaufs, ja er war auch noch in seinem Irrtum bedeutend, aber wegen seiner schlechten Presse hat sich die Nachwelt nur wenig mit ihm befaßt. Auch sind Riolans Argumente, abgesehen vom historischen Aspekt, ein interessanter Beitrag zur Psychologie des Irrtums. Wir wollen daher einigen von seinen Einwänden und Begründungen nachgehen.

Die Quellen zu dem Streit zwischen Riolan und Schlegel sind vor allen Dingen drei Publikationen ${ }^{6,7}$, nämlich zunächst Riolans (= I) Instauratio magna, eine Ablehnung von Harveys Lehre in seinen Opuscula Anatomica Nova, London 1649. Sie veranlaßte Schlegel zu seiner Angriffsschrift gegen Riolan mit dem Titel De Sanguinis Motu Commentatio, in qua praecipue

${ }^{4}$ Insbesondere des Nachweises der Kapillaren (MaLPighi, 1661). Vgl. K. E. RothschuH, Die Entwicklung der Kreislauflehre im Anschluß an William Harvey, Klin. Wschr. 35 (1957) 605-12.

5 Thomas Bartholinus (1616-1680), Anatomia ... tertium ad sanguinis circulationem reformata, Lugd. Batav. 1651.

${ }^{6}$ Die erste kurze Ablehnung von Harveys Kreislauf findet sich in Riolans Encheiridium anatomicum et pathologicum ... ad usum theatri anatomici adornatum, Parisiis 1648, Lugd. Batav. 1649, S. 220.

${ }^{7}$ Die beiden Hauptquellen sind im folgenden mit I und II zitiert: I. Joanne Riolano Authore. Opuscula Anatomica Nova, quae nunc primum in lucem prodeunt. Instauratio magna physicae et medicinae per novam doctrinam de motu circulario sanguinis in corde. Accessere notae in J.Wallaei duas epistulas de circulatione sanguinis, Londini 1649; II. Joannis Riolani Opuscula varia et nova in primis de motu sanguinis, ejusque circulatione vera, ex doctrina Hippocratis, Parisiis 1652. Darin befindet sich die Abhandlung Riolans, Defensio adversum inquisitionem Pauli Marquardi Slegelii, Medici Hamburgensis, de sanguinis motu (S. 213-356). 


\title{
PAULI MARQUARTI SLEGELII Med. Hamburgenfis,
}

DE

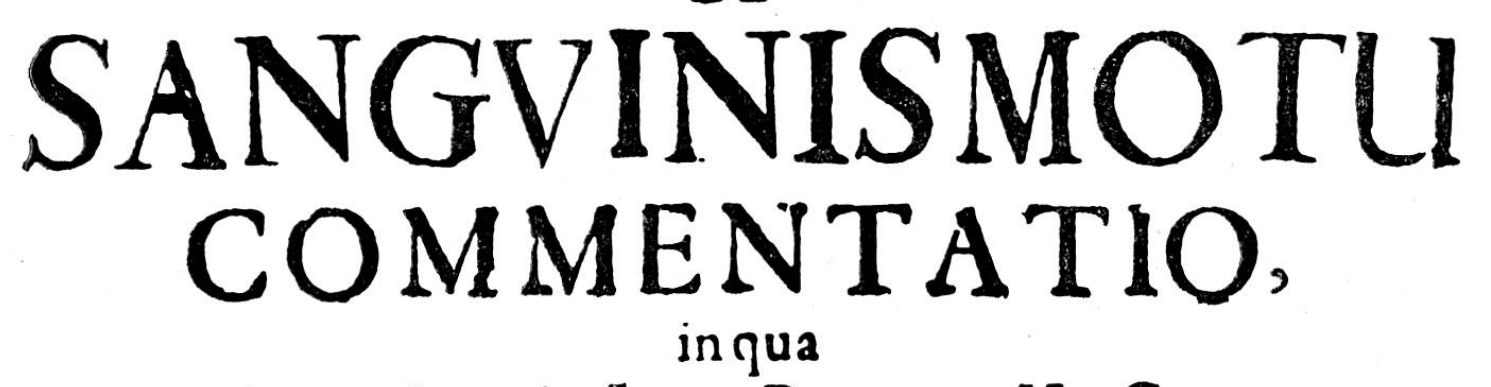

procipue in Joh. Riolani, V. C.

fencentiam inquiritur,

\author{
ad \\ Clarif: Viros, \\ D. D.CHRISTOPH. SCHELHAM- \\ MERUM
}

Anatom. Chirurgix, \& Botanic.

P.P. in illuftri Salana,
\&
D. D. GILIANUM GARTZIUM,
Medicum Hamburgenfem,
Anatomicos exercitatifimos.

\section{HAMBURGI, \\ Typis Jacobi Rabenline, fumptu Zach. Hertelii, Bibliob. Hamb. \\ A. C. slo. bos L.}

Abb. 1. Das Titelblatt der Streitschrift vom Jahre 1650, in der Paul M. Schlegel (Hamburg) die Kreislauflehre Harveys gegen die Kritik von Jean Riolan jun. (Paris) verteidigte 


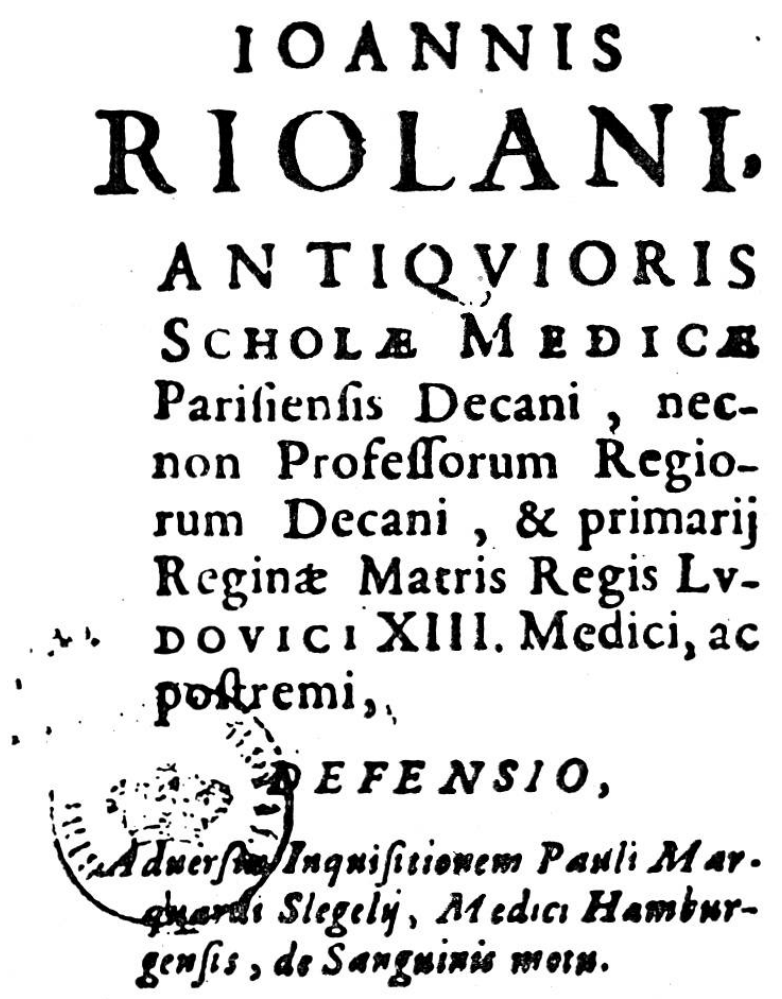

T iii)

Abb. 2. Das Titelblatt der Schrift, mit der Jean Riolan jun. (Paris) im Jahre 1652 SchleGELS Angriff beantwortete. Sie ist ein Teil des Opuscula varia et nova ... von RiolaN

in Joh. Riolani V. C. sententiam inquiritur (1650) (Abb. 1). Auf diesen Angriff antwortete Riolan zwei Jahre später mit einer Abhandlung (= II) Defensio adversus inquisitionem P. M. Slegelii (Paris 1652) (Abb. 2). Wir betrachten im folgenden diesen Meinungsstreit weniger von der Seite Schlegels als vielmehr im Hinblick auf Riolans Gegenargumente, denn erst dann wird man verstehen, warum auch neben ihm andere bedeutende Zeitgenossen wie Caspar Hofmann (1572-1648) - übrigens ein Lehrer Schlegels weiterhin an der alten Galenschen Auffassung festgehalten haben ${ }^{8}$.

${ }^{8}$ Vgl. dazu E.V. Ferrario, F.N.L. Poynter und K.J. Franklin, William Harveys Debate with Caspar Hofmann on the Circulation of the Blood, J. Hist. Med. All. Sci. 15 (1960) 7-21. 


\section{Biographisches zu Schlegel und Riolan}

Jean Riolan jun. (Abb. 3) wurde geboren zu Paris im Jahre 1580 als Sohn des berühmten Anatomen und Arztes Jean Riolan sen. Er promovierte 1604 in Paris und wurde dort 1613 Professor für Anatomie und Botanik. Riolan jun. verfaßte eine große Zahl von Schriften zu allen möglichen Fragen der Anatomie und Medizin, er beschrieb vor allen Dingen das Netz, den Bau des Mesenteriums, des Dickdarms, die Samenkanälchen und war Leibarzt Heinrichs IV. und Ludwigs XIII. Unter seinen bekannteren Werken nennen wir das Encheiridium anatomicum et pathologicum (Paris 1648 und Leiden 1649), ferner die Opuscula anatomica nova (London 1649). Riolan hat sich in weiteren Streitschriften gegen die neueren Entdeckungen einiger Anatomen seiner Zeit, z.B. gegen Bartholinus, gegen Pecquet und Walaeus gewandt ${ }^{9}$. Er starb 1657, einige Jahre nach Schlegel.

Paul Marquart Schlegel (Abb. 4) wurde 1605 in Hamburg geboren ${ }^{10}$, begann 1626 sein Medizinstudium in Altdorf, unter anderem bei Caspar Hofmann. Dann ging er 1627 zu Werner Rolfinck nach Wittenberg, später Jena. Rolfinck, seinerseits ebenfalls ein Hamburger, hat seinen Namen auch durch hervorragende anatomische Schriften und durch sein frühes Eintreten für den Blutkreislauf bekannt gemacht. Schlegel befand sich ab 1631 sechs Jahre auf einer Peregrinatio bis 1638, zunächst durch Holland und England. Er arbeitete dann zwei Jahre bei Riolan. Über Montpellier und Lyon kam er nach Padua, wo er 1636 promovierte. Zurückgekehrt, hielt er sich noch einmal (1638) vier Monate lang bei C. Hofmann auf. Im Jahre 1639 wurde er neben Rolfinck Professor der Anatomie, der Botanik und Chirurgie in Jena, 1640-41 wurde er Rektor der Universität. Auch war er Leibarzt des Herzogs von Sachsen. Im Herbst 1642 verließ er Jena und nahm eine Stelle als Stadtphysikus in Hamburg an. Seine Haupttätigkeit bestand hier in der Sorge um das öffentliche Gesundheitswesen. Er errichtete auch eine anatomische Lehranstalt für Chirurgen und führte eigene Sektionen durch, gegen die sich mancherlei Widerspruch unter seinen Mitbürgern erhob. Er starb 1653 an einer Seuche. Ob er die Antwortschrift von Riolan (II) noch vor seinem Tode erhalten hat, ist unbekannt ${ }^{11}$.

${ }^{9}$ Vgl. K.E. Roтнsснuн, Geschichte der Physiologie, Berlin/Heidelberg 1953.

${ }^{10}$ Biographisches in Nikolaus Wilkens, Hamburgischer Ehrentempel, Hamburg 1770, ferner in Giese/v. HaGen, Geschichte der medizinischen Fakultät Jena, Jena 1958.

11 Harvey bedankte sich bei Schlegel für die Verteidigungsschrift in einem freundlichen und sachlich interessanten Brief vom 26. März 1651. Vgl. K.F. Franklin, William Harvey, Englishman, London 1961, S. 116. 


\section{Die Argumente Riolans gegen die Harveysche Kreislauflehre}

Da wir hier die Harveysche Kreislauflehre in ihrer Fassung vom Jahre 1628 als bekannt voraussetzen können, genügt es, die bedeutsamsten Argumente Riolans gegen einige Hauptpunkte der Harvey-Schlegelschen Lehre anzuführen ${ }^{12}$.

\section{a) Anatomisch-physiologische Argumente gegen Harveys Kreislauflehre}

Nach Harvey-Schlegel bringen die Arterien nährendes, im Herzen aufgewärmtes und mit Spiritus angereichertes Blut zur Peripherie, dieses Blut kehrt an Nahrung, Wärme und Spiritus verarmt über die Venen zum rechten Herzen zurück. Riolan leugnet diesen großen Kreislauf; er läßt weder eine Lungenpassage noch einen arteriovenösen Übertritt von Blut im Gewebe zu, vertritt aber einen kurzen Kreislauf, der darin besteht, daß durch feine Anastomosen zwischen der Aorta und der Vena cava etwas spirituelles Blut über die Venen zum Herzen zurückkehrt (II/254, 259, 295). Sonst gelangt in den Arterien belebendes warmes Blut tropfenweise zur Peripherie, wo es verbraucht wird; die Venen transportieren tropfenweise ernährendes Blut in die Gewebe, wo es ebenfalls verbraucht wird (Abb. 5).

Harveys Ermittlung des Schlagvolumens und der Schlagzahl am eröffneten tierischen Herzen, seine Berechnung der zirkulierenden Blutmenge und der Kreislaufzeit lehnt Riolan mit folgender Begründung ab: Tierversuche entstellen die Wahrheit; denn am sterbenden Tier, also unter nicht normalen Verhältnissen, strebt alles Blut zu seiner Quelle, dem Herzen, zurück (II/249). Normalerweise wirft das Herz höchstens 1 bis 2 Tropfen pro Schlag aus, denn mehr braucht es nicht für seine Ernährung und die Erhaltung des Schlagvermögens (Vis pulsifica). Nimmt man nämlich das Herz eines Tieres aus dem Körper heraus, so schlägt es mit dem wenigen darin verbleibenden Blut noch eine ganze Zeit weiter. Größere Blutmengen sind überflüssig. Daher ist anzunehmen, daß das arterielle Blut nur tropfenweise über die Arterien verteilt wird, ja daß viele Schläge des Herzens ohne Blutauswurf erfolgen. Wenn das stimmt, ist Harveys Berechnung falsch (II/326, 327). Falsch ist daher auch seine Auffassung, daß der arterielle

12 Mein Mitarbeiter Prieshof hat die Argumente von Schlegel denen von Riolan Punkt für Punkt gegenübergestellt. Hier kam es mehr darauf an, zu zeigen, daß Riolan mit oft guten Überlegungen gegen Schlegel bzw. Harvey argumentierte. Vgl. JoH. Dietr. Prieshof, Der Streit um den Blutkreislauf zwischen Johannes Riolan und Paul Marquart Schlegel um 1650, Inaug. Diss. Med. Münster (Westfalen) 1961, 51 Seiten, Maschinenschrift. 


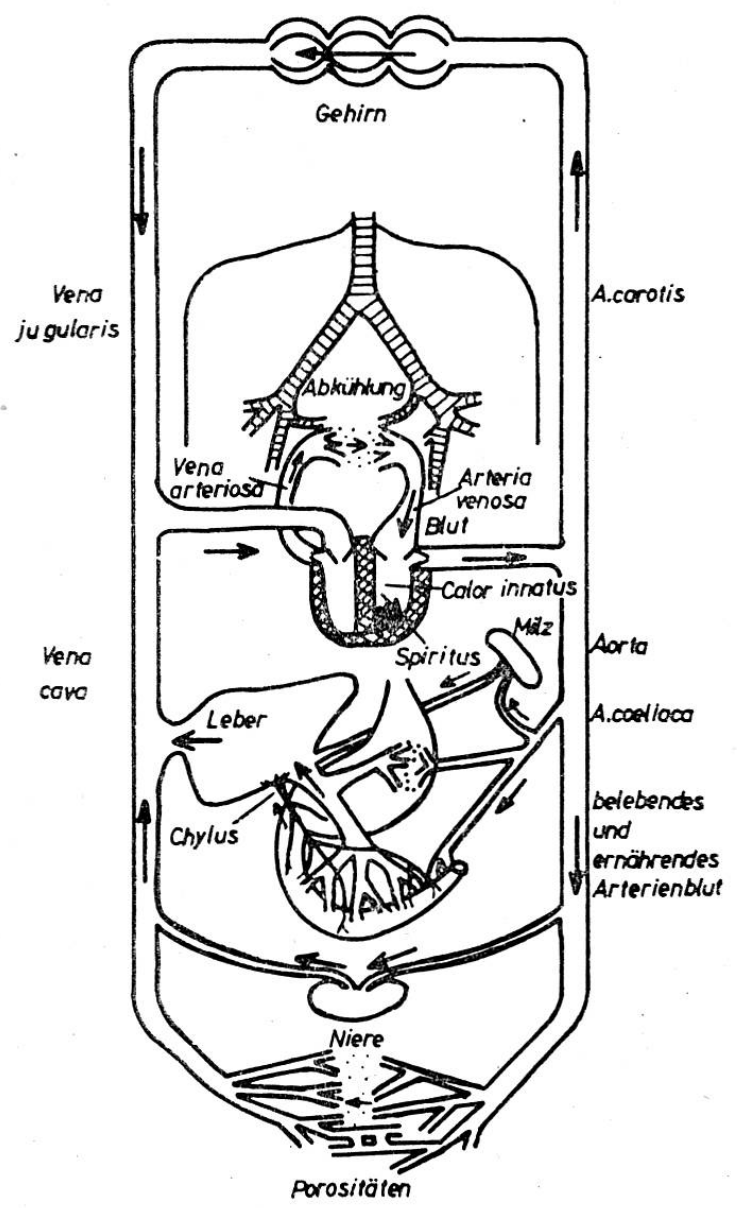

nach P.M. Schlegel 1650

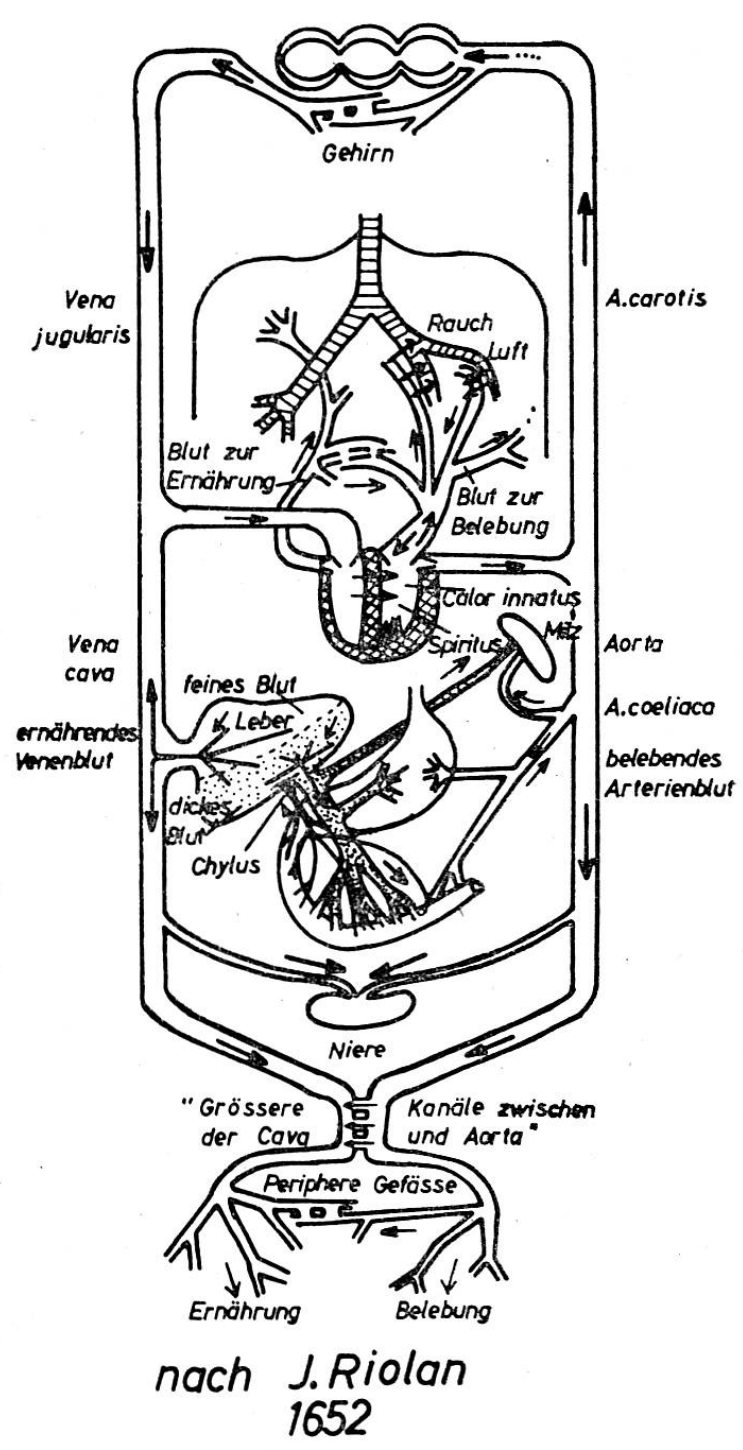

Abb. 5. Versuch einer graphischen Darstellung der Kreislauflehre bei ScHLEgel (links) und der Blutbewegungslehre von Riolan (rechts). Bei HARveY und Schlegel fließt das Blut im Pfortadersystem und in den übrigen Venen stets zum Herzen, passiert die Lunge durch angenommene Porositäten, verläßt die linke Kammer erwärmt und mit Spiritus vitalis «vitalisiert», dann gelangt es über die arteriellen Gefäße in die Peripherie. Dort passiert es durch unbekannte Wege herüber zu den kleinen Venen und fließt zum Herzen zurück. Nach Riolan gelangen aus dem Darm und über die Leber nur die feinsten Anteile des Blutes in die Vena cava. Sie fließen hier zum größten Teil in Richtung zur Peripherie, wo sie tropfenweise anlangen und verbraucht werden. Der kleinere Teil des Cavablutes fließt zum Herzen, vornehmlich zur Erhaltung der Vis pulsifica. Seine feinsten Bestandteile treten durch die Poren der Herzscheidewand in die linke Kammer hinüber, ein anderer Teil gelangt zur Ernährung in die Lungen. Das in der linken Kammer erwärmte und mit Spiritus angereicherte Blut fließt in den Arterien tropfenweise zur Peripherie, wo es zur Belebung der Teile verbraucht wird. Ein ganz kleiner Teil des arteriellen Blutes geht im Rumpfgebiet durch kleinste Verbindungen von der Aorta direkt in die Vena cava über (unten im rechten Bild). In der Vena portae fließt der gröbere Anteil des Blutes in die Darmgefäße zur Ablagerung peripherwärts ab 
Puls durch die Anfüllung der Arterien mit Blut entsteht, sie pulsieren aus eigenem Vermögen (II/327). Handelt es sich aber nur um einige Tropfen Blut bei jedem Herzschlag, so entfallen auch alle Bedenken gegen die Septumpassage. Das Septum ist oben dünn, und es besitzt Spalten. Die Nieren sind doch noch viel dichter als das Septum, und trotzdem fließt der Harn hindurch (II/283). Eine Passage durch die Lungen ist also überflüssig. Nur unter den widernatürlichen Verhältnissen des Tierversuchs ist das anders (II/221, 229). Warum soll auch soviel Blut durch die Lungen fließen? Es würde nur die Atmung erschweren (II/272), und das Blut würde sich zu sehr abkühlen, bevor es in der linken Kammer wieder erwärmt wird (II/284). Schließlich bestünde Gefahr, daß dauernd, besonders bei jedem Hustenstoß, Blut aus den Porositäten zwischen den Lungengefäßen und den Atemwegen heraustreten würde, durch die normalerweise die Unreinigkeiten des Blutes (als Schleim) abgegeben werden (II/275, 279). Da sich ferner die Bewegungen des Herzens und die Atembewegungen nicht gleichzeitig vollziehen, so kann man sich einen Blutdurchfluß durch die Lungen im Sinne Harveys gar nicht vorstellen. (II/277), das Blut müßte sich während der Ausatmung jeweils anstauen (II/276ff.). Auch findet man bei Sektionen die Lungen meist blutleer (II/274). Bei Stichverletzungen der Lungen fließt trotz starker Verletzungen durchaus nicht alles Blut heraus (II/288). Wenn Harvey-Schlegel meinen, daß die Vielheit gleichzeitiger Vorgänge im engen Gefäßrohr der Vena arteriosa unglaubwürdig sei (Luft zum Herzen, Ruß und belebendes Blut zur Lunge), so hält Riolan das nicht für problematisch (II/288), da es sich nur um wenige Tropfen, nicht aber um ein größeres Blutvolumen mit hoher Geschwindigkeit handelt. Die schnelle Blutströmung, welche die Harveyaner annehmen, ist überhaupt sinnlos, denn je schneller das Blut fließt, desto weniger hat es Zeit, die Ernährung, Belebung und Erwärmung der Peripherie zu vollziehen. Auch würde dadurch die Absonderung der Spiritus animales im Gehirn gefährdet (II/330). Ja das Blut würde seine Wärme nur ungenügend abgeben können und viel zu hei $\beta$ werden (I/58). Warum soll überhaupt das venöse Blut zum Herzen zurückfließen? Cui bono? Schon Hippokrates lehrte die nährende Aufgabe der Venen; diese und die Organe ziehen die nährende Materie an sich (II/309). Bei der Sektion einer Schwangeren wird das offenkundig, denn hier ziehen dreimal dickere Venen als normalerweise zum Uterus und doch wohl zur Ernährung des Fötus (II/300).

Wenn Harvey und Schlegel aus Tierversuchen schließen (II/306), das Blut fließe in der Vena portae zur Leber und weiter herzwärts, so irren sie 
wieder aus dem schon erwähnten Grunde. Warum sollte auch das Blut der Vena portae zum Herzen fließen? Sie führt dickes unreines, an «Küchenabfällen » reiches Blut, das in die Bauchorgane fließt. Nur die reinsten Bestandteile werden in der Leber ausgesondert und gehen in die Vena cava über (II/307), um dann teils zentralwärts zum Herzen, teils peripherwärts zu den Gliedern zu fließen. Nur in den Jugularvenen fließt überschüssiges Blut vom Gehirn abwärts; denn das Gehirn als kühles Organ braucht zur Erzeugung der Spiritus animales vornehmlich das spirituelle Blut der Arterien. Dieses darf aber nicht zu schnell fließen, denn eine zu heftige Blutströmung im Gehirn würde u.U. die gebildeten Spiritus animales mitreißen (II/329, 330); daher ist der Abfluß des überschüssigen Blutes über die Jugularvenen (II/316, 318) notwendig.

\section{b) Klinische Argumente Riolans gegen die Kreislauflehre}

Wenn so viel Blut so schnell durch die Gefäße fließt, wie die Kreislaufanhänger meinen, dann würde eine Stauung und Stockung von Blut in kranken Organen unmöglich sein (II/334), doch ist das eine alltägliche Erfahrungstatsache. Schlegel scheint sich zu wenig praktisch zu betätigen, wenn er das übersieht (II/311, 312). Wie sollte sich ferner vier Tage lang das unreine Menstrualslut ansammeln, bevor es in der Regelblutung abgegeben wird (II/329)? Wie soll die Milch in der Brustdrüse aus dem Blut abgesondert werden, wenn das nährende Blut dort nicht verweilen kann (II/329)? Jeder Arzt kennt die Gefährlichkeit eines Rückflusses von Hämorrhoidalblut; das soll aber nach der Kreislauflehre in den Venen dauernd passieren (II/32). Die Indikationen zur Blutausleerung verlieren weitgehend ihren

Sinn, denn was soll eine derivatio am Orte der Blutanhäufung und Entzündung, wenn das Angesammelte sogleich ersetzt wird? Was soll dann noch eine revulsio, also ein Abziehen von Blut durch Aderlaß an entfernter Stelle, z.B. bei Plethora des Gehirns am Fuß (I/59)? Wenn die Kreislauflehre stimmt, dann müßte auch eine Krankheitsmaterie, z. B. das Luesgift, sofort in den ganzen Körper verteilt werden. Warum läßt aber der Ausbruch der Krankheit so lange auf sich warten (I/121) ? Wir geben ferner Medikamente für ganz bestimmte Organe. Wie sollten sie dort verbleiben und wirken, wenn der Kreislauf sie sogleich wieder entführt (II/342) ? Wie soll man den häufigen Befund der Polypen (Gerinnsel) im rechten Herzen verstehen, wenn nicht dadurch, daß das Septum eine unreine Materie vor dem Ubergang in die linke Kammer bewahrt hat (II/285). Überhaupt sind die ganzen Lehren der Neuerer der praktischen Medizin verderblich. «Wenn 
die Leber nicht primärer Teil, nicht Sitz der facultas naturalis, nicht Bildner des Blutes ist ..., nicht die Galle des Pfortaderblutes ausscheidet, dann hängen von ihr nicht mehr jene der Leber zugewiesenen Krankheiten ab wie Retention des Chylus, ungenügende Blutbildung, Diarrhoea hepatica, Cachexia, Atrophia, Hydrops. Bei der Behandlung dieser Leiden wäre nicht mehr auf die Leber Rücksicht zu nehmen, keine darauf zielenden Mittel anzuwenden, Leberkrankheiten wären nicht für die Corruption der Blutmasse verantwortlich zu machen. Augenscheinlich hat also Hippokrates halluziniert, als er die Gesundheit und Integrität der Organe einer gesunden Leber zuschrieb»(I/177). Die Blutbildung in der Leber leugnen, heißt «totam medicinam pertubare et confundere» (I/120).

\section{Beurteilung und Analyse der Argumente des Riolan gegen die Kreislauflehre}

Man wird sich nach dieser Aufzählung nicht des Eindrucks erwehren können, daß die Argumente Riolans gegen die Kreislauflehre auf die Ärzte seiner Zeit erheblichen Eindruck machen mußten. Er übersieht die Konsequenzen von Harveys Lehre wohl wie kaum ein anderer und versuchte, die Lehren der großen Meister Aristoteles, Hippokrates und Galen und damit die alte überlieferte Heilkunde zu retten. Ja, er versteigt sich zu dem Satz: «Imo nihil novi sub sole hodie reperitur» (Praefatio I/4).

Der schwerwiegendste Fehler des Riolan war die Ablehnung des Tierversuches für die Aufklärung der menschlichen Physiologie. Gewiß hatte er recht, wenn er sagte, daß bei den Tieren in der Körperhaltung, der Lage und dem Bau des Herzens und anderer Organe sowie der Fellbedeckung des Körpers andere Verhältnisse vorliegen (II/345, 346), aber er ging darin zu weit, wenn er sagte: «Ista experimenta sunt mihi valde suspecta» (I/93).

Riolans zweiter Hauptfehler aber ist seine dauernde Frage «cui bono?» Er fragt zunächst stets nach dem Nutzen eines Organs, eines Vorgangs, und zwar stets innerhalb des alten Systems der Physiologie von Galen. Was dazu nicht paßt, wird nicht anerkannt. So hatte Harvey recht, als er in seinem zweiten Brief an Riolan (1649) ${ }^{13}$ sagte: «Es ist unsere erste Pflicht

13 Der zweite Brief Harveys an Riolan (1649) ist wohl das kühnste und revolutionierendste, was HARveY je schrieb. Er bezeichnet die Spiritus als refugium der Unwissenheit, versucht ihre Nichtexistenz experimentell (Eröffnen einer Arterie unter Wasser) zu beweisen, verlegt die Herkunft der Körperwärme in das Blut, widerlegt experimentell die vis pulsifica der Arterien, entwickelt eine Pathologie des Kreislaufs, usw. Dieser Aufsatz bringt ihm Riolans Urteil ein: Harvey ridiculus (I/135), audax (I/136). 
zu fragen, ob ein Ding ist oder nicht ist, bevor man fragt, wozu es da ist.» Wer aber hatte damals schon jene unvoreingenommene, induktive Art des Vorgehens wie Harvey ? ${ }^{14}$ Riolans Grundhaltung ist philosophisch-deduktivsystematisch; er bezweifelt nicht die Richtigkeit der überlieferten Prinzipien und Ausgangssatzungen; er beurteilt das Neue vorwiegend logisch, ob es sich mit den alten Satzungen verträgt, nicht aber empirisch-induktiv als Phänomen. Harvey und Schlegel durchdenken die kausalen dynamischen Ursache-Wirkungs-Zusammenhänge, die sich aus den neuen Befunden für die Auffassung der Organfunktionen ergeben, Riolan durchdenkt die logischen Konsequenzen der neuen Lehren für die alte Medizin. So stehen Harvey und Riolan einander gegenüber als Vertreter der alten und der neuen Zeit, Harvey als Sensualist, Riolan als theoretischer Systematiker.

Versuchen wir abschließend die Irrtümer Riolans unter die möglichen Arten des Irrtums in den Erfahrungswissenschaften einzuordnen: Wenn Wissenschaft in diesem Sinne die systematische Übertragung von verifizierten Erfahrungssachverhalten auf Urteile und Aussagen darstellt, dann gibt es zwei große Gruppen von Fehlern bzw. Irrtümern: 1. solche bei der Ermittlung der Erfahrungssachverhalte durch Beobachtung und Experiment und 2. solche bei der Beurteilung und Generalisierung der Sachverhalte. Riolan war unfähig zu einer unvoreingenommenen Beobachtung. Seine Vorurteile gestatteten ihm weder eine unbefangene Zuwendung zum Beobachtbaren, noch ließen sie ihm die Grenzen von Beobachtung und theoretischer Interpretation sichtbar werden. Es waren sogar dreierlei Arten der Urteils-Voreingenommenheit, die ihn hinderten, die neue Kreislauflehre anzuerkennen, nämlich erstens ein autoritatives Vorurteil durch seinen Galenismus, zweitens ein systematisches Vorurteil, gebildet durch die Logik des von ihm vertretenen Gesamtsystems der Physiologie und Heilkunde und drittens ein affektives Vorurteil, erwachsen aus verletzter Eitelkeit und persönlicher Abneigung. Trotzdem sollte man es aufgeben, seinen Widerstand gegen die Kreislauflehre nur als Ausfluß von Niedertracht und Dummheit zu bewerten. Man wird damit weder der historischen Situation noch dem Range Riolans gerecht.

${ }^{14}$ Harvey steht dem Lockeschen Sensualismus nahe: In seinem zweiten Brief an RiolaN 1649 heißt es: «Denique hoc est, quod enarrare \& patefacere, per observationes \& experimenta conabar, non ex causis \& principis probabilibus demonstrare, sed, per sensum \& experientiam, confirmatam reddere, anatomico more, tamquam majori authoritate, volui » (nach Bibl. Anatomica, von D. Le Clerc und I. Jac. Mangetus, Tom. II, Genevae 1685, S. 77). 


\section{Rousseau et Buffon}

\section{Par Jean Starobinski*}

Rousseau, dans le Discours de l'Inégalité, et surtout dans les notes de ce texte, a emprunté très ouvertement à l'Histoire Naturelle. Sur toutes les questions de science, c'est l'autorité de Buffon que le Discours allègue constamment, avec une admiration qui ne craint pas de s'avouer: «Dès mon premier pas je m'appuie avec confiance sur une de ces autorités respectables pour les philosophes, parce qu'elles viennent d'une raison solide et sublime, qu'eux seuls savent trouver et sentir.» ${ }^{1}$

Assurément, tout paraît dissemblable à première vue, si l'on entreprend de comparer l'intention qui anime l'œuvre de Rousseau et le dessein descriptif de Buffon. Rousseau, c'est l'esprit révolté qui se manifeste par le refus, par la négativité; quand il parle de la nature, c'est pour l'opposer à la société policée de son temps: la notion de nature est une arme critique contre les valeurs admises par la société. Buffon, au contraire, pose la nature sans l'opposer dramatiquement à la culture. Il sait que l'art humain altère la nature, fait «dégénérer» les animaux, mais il y voit une confirmation des pouvoirs souverains de la raison. Il dirige son regard vers un monde infiniment varié, dont la diversité ne sera pas rebelle à la patience de l'observateur. L'univers offre un spectacle satisfaisant: point de faille ni de conflit. Pour vaste qu'il soit, le réel se laisse décrire; l'intelligence saura discerner les nuances et les gradations à travers lesquelles l'échelle des êtres se développe des minéraux jusqu'à l'homme; autour de la figure prépondérante de l'homme raisonnable, le langage déploiera avec ordre et clarté un inventaire complet de la richesse du monde visible. Les tempéraments, les œuvres sont trop différents pour qu'on ait jugé opportun de confronter, sur le fond, la pensée de Jean-Jacques et celle de Buffon. La tâche en vaut cependant la peine $^{2}$, car Rousseau n'a pas seulement cherché dans l'Histoire Naturelle

*Dr.-Markus-Guggenheim-Vortrag, gehalten an der Tagung der Schweizerischen Naturforschenden Gesellschaft in der Sektion für Geschichte der Medizin und der Naturwissenschaften, Sitten, den 31. August 1963.

1 J.-J. Rousseau, Euvres complètes, édition Hachette 1865, t. I, p. 127 (orthographe modernisée).

2 Il faut savoir gré à Jean Morel (Recherches sur les sources du Discours de l'Inégalité, Annales Jean-Jacques Rousseau, V, 1909, p. 119-98), et surtout M. OTIS Fellows (Buffon and Rousseau: Aspects of relationship, PMLA, juin 1960, p. 184-96) d'avoir indiqué les points de rencontre les plus importants. 
tout un arsenal de faits et de preuves à l'appui de ses propres théories (sur l'importance du toucher, sur la longévité du cheval, sur la durée de la vie, sur l'alimentation, sur l'emmaillottement, etc...); il y a trouvé une image de l'homme ou, si l'on préfère, une anthropologie philosophique, qu'il a pu accepter en grande partie et qu'il a contredite en quelques points importants. L'essentiel est là, et c'est dans leurs vues sur la condition humaine qu'il faut comparer Rousseau et Buffon, pour relever les ressemblances et les divergences.

Il est impossible de s'y méprendre. Dès la première phrase de la préface, une note nous avertit que Rousseau a sous les yeux l'Histoire Naturelle de l'Homme. On lit chez Rousseau: «La plus utile et la moins avancée de toutes les connaissances humaines me paraît celle de l'homme.» ${ }^{3}$ Et il nous renvoie à un assez long passage de Buffon: «Quelqu'intérêt que nous ayons à nous connaître nous-mêmes, je ne sais si nous ne connaissons pas mieux tout ce qui n'est pas nous... $»^{4}$ Le citoyen de Genève, au moment où il entreprend «l'étude historique de la morale», est heureux de trouver un répondant en la personne du grand naturaliste; tous deux constatent que la science de l'homme fait encore défaut dans le système des connaissances exactes. Bien que Buffon s'arrête au seuil des problèmes de la vie sociale, l'Histoire Naturelle de l'Homme constitue pour Rousseau un précédent particulièrement précieux. Il y trouve en effet une étude naturaliste de la condition humaine, d'où, par précaution de méthode, toute considération théologique est exclue; de plus, le long chapitre sur les «Variétés dans l'espèce humaine» élargit singulièrement l'horizon et invite à interpréter historiquement la cause des différences physiques actuellement constatées: «Tout concourt... à prouver que le genre humain n'est pas composé d'espèces essentiellement différentes entre elles, qu'au contraire il n'y a eu originairement qu'une seule espèce d'hommes qui s'étant multipliée et répandue sur toute la surface de la terre, a subi différents changements par l'influence du climat, par la différence de la nourriture, par celle de la manière de vivre, par les maladies épidémiques, et aussi par le mélange varié à l'infini des individus plus ou moins ressemblants. ${ }^{5}$ Voilà qui ne pouvait manquer d'être relevé par un esprit désireux de prouver que le système social des Européens civilisés n'est ni le seul, ni le meilleur et qu'il est le

${ }^{3}$ J.-J. Rousseau, CEuvres complètes, Hachette, 1865, t. I, p. 78.

${ }^{4}$ Ibid., cf. Buffon, Euvres complètes, édition Flourens, Paris, Garnier, s.d., t. II, p. 1.

${ }^{5}$ Buffon, Euvres complètes, édition Flourens, Paris, Garnier, s. d., t. II, p. 221 (toutes les citations de Buffon renvoient à la même édition). 
produit d'une histoire corruptrice. Quand Rousseau parle de la difficulté que nous éprouvons à démêler l'originaire et le factice, il aura été précédé et guidé par Buffon: «L'homme sauvage est, de tous les animaux le plus singulier, le moins connu et le plus difficile à décrire. Mais nous distinguons si peu ce que la Nature seule nous a donné de ce que l'éducation, l'art et l'exemple nous ont communiqué, ou nous le confondons si bien, qu'il ne serait pas étonnant que nous méconnussions totalement au portrait d'un sauvage, s'il nous était présenté avec les vraies couleurs et les seuls traits naturels qui doivent en faire le caractère.

«Un sauvage absolument sauvage... (serait) un spectacle curieux pour le philosophe, il pourrait en observant son sauvage évaluer au juste la force des appétits de la Nature, il y verrait l'âme à découvert, il en distinguerait tous les mouvements naturels, et peut-être y reconnaîtrait-il plus de douceur, de tranquillité et de calme dans la sienne, peut-être verrait-il clairement que la vertu appartient à l'homme sauvage plus qu'à l'homme civilisé, et que le vice n'a pris naissance que dans la société. ${ }^{6}$

C'est là, en raccourci, la définition de l'homme de la nature selon Rousseau (qui substituerait toutefois l'idée de bonté à celle de vertu). Avec de tels précédents et avec pareille caution, comment ne pas aller hardiment de l'avant? Rousseau a pu trouver dans l'impudeur scientifique de Buffon un encouragement à l'audace, de même que les hypothèses de la Théorie de la Terre l'encourageaient à recourir à son tour aux «raisonnements hypothétiques et conditionnels... semblables à ceux que font tous les jours nos physiciens sur la formation du monde. $)^{7}$ Buffon proteste de son respect pour l'autorité des faits, mais il a passé, à juste titre, comme Rousseau, pour un homme à hypothèses. Ils ont dû faire face aux mêmes reproches, et souvent ils ont été critiqués conjointement. On lit en effet,' en août 1756, sous la plume de Formey: «M. Rousseau est assez dans son genre ce que M. de Buffon est dans le sien; il manie les hommes comme ce philosophe manie la Nature et l'Univers; il fait des hypothèses sur la Société comme l'Académicien en fait sur les Globes de l'Univers et l'origine des Planètes. $)^{8}$

Il ne faut toutefois pas se laisser égarer par les affirmations de Formey. S'il y a une analogie entre la méthode de Rousseau et celle de Buffon, cette

${ }^{6}$ Buffon, t. II, p. $200 \mathrm{f}$.

${ }^{7}$ Rousseau, Euvres complètes, édition Hachette, t. I, p. 83.

${ }^{8}$ Bibliothèque impartiale, pour les mois de Juillet et Août 1756, t. XIV, première partie, Göttingue/Leyde 1756, p. 62. 
ressemblance réside moins dans le recours à l'hypothèse que dans le parti qu'ils prennent l'un et l'autre de commencer par définir exhaustivement une forme élémentaire d'existence, afin de mieux apercevoir, par contraste, ce qui relève d'une faculté supérieure ou d'un développement ultérieur. Dans le Discours sur la Nature des Animaux, conformément à un dualisme hérité de Descartes et peut-être maintenu pour la commodité de l'exposé, Buffon se propose de décrire au plus juste les opérations dont est capable la matière organisée, par la seule vertu des lois mécaniques de la nature. On sait que, pour Buffon comme pour Descartes, la vie ne comporte aucun privilège particulier: «Le vivant et l'animé, au lieu d'être un degré métaphysique des êtres, est une propriété physique de la matière.» ${ }^{9}$ La grande frontière métaphysique intervient entre la mécanique matérielle du corps vivant et l'activité de l'âme raisonnable. Cette frontière passe en nous, puisque nous sommes tout ensemble corps et esprit, matière animée et substance pensante. Nous ne connaîtrons avec certitude ce qui fait l'humanité de l'homme qu'à la condition de savoir où s'arrêtent les pouvoirs liés à son animalité. D'où l'utilité d'une étude àttentive de tout ce qui relève du sens intime matériel: "Voyons ce que ce sens intérieur matériel peut produire: lorsque nous aurons fixé l'étendue de la sphère de son activité, tout ce qui n'y sera pas compris dépendra nécessairement du sens spirituel: l'âme fera tout ce que ce sens matériel ne peut faire. Si nous établissons des limites certaines entre ces deux puissances, nous reconnaitrons clairement ce qui appartient à chacune; nous distinguerons clairement ce que les animaux ont de commun avec nous, et ce que nous avons au-dessus d'eux. ${ }^{10}$ Qu'observerons-nous? Que l'homme seul est capable de juger, c'est-à-dire de comparer; qu'il est seul capable de prévoir et de se souvenir. Et, tandis que dans leurs activités les plus ingénieuses les animaux obéissent immuablement à leur instinct, répétant les mêmes actions sans être capables de les modifier, l'homme possède un pouvoir de perfectionnement et de progrès qui n'appartient qu'à lui seul: «Si [les animaux] étaient doués de la puissance de réfléchir, même au plus petit degré, ils seraient capables de quelque espèce de progrès, ils acquerraient plus d'industrie. Ils n'inventent rien, ils ne perfectionnent rien, ils ne réfléchissent par conséquent sur rien, ils ne font jamais que les mêmes choses de la même façon. " ${ }^{11} \mathrm{Il}$ n'y a rien en tout

\footnotetext{
${ }^{9}$ Buffon, t. I, p. 434.

${ }^{10}$ Buffon, t. II, p. $327 \mathrm{f}$.

11 Buffon, t. II, p. 7.
} 
ceci qui ne se retrouve dans le second Discours ou dans la Profession de Foi. Non certes que Rousseau ait tout emprunté à Buffon, mais là où Rousseau ne doit rien à Buffon, l'on constate que tous deux puissent aux mêmes sources et se rattachent au même cartésianisme modifié par LocKE. Certaines nuances toutefois méritent d'être relevées, car elles ne sont pas sans conséquence: pour Buffon, la spiritualité de l'homme réside dans son entendement; pour Rousseau, elle consiste essentiellement dans la liberté. Quoi qu'il en soit, quand Rousseau affirme que la perfectibilité est l'apanage de l'homme, il trouve des lecteurs que la lecture de Buffon a suffisamment avertis pour que ce néologisme ne les surprenne pas.

Buffon n'est pas ouvertement transformiste; s'il admet une certaine évolution, c'est sous la forme de la dégénérescence, et dans un cadre restreint, à l'intérieur de certaines espèces que la domestication modifie. Dans l'important chapitre sur l'Ane, Buffon a soulevé l'hypothèse d'une grande famille des êtres vivants où les espèces surgiraient les unes à partir des autres; mais cette hypothèse est rejetée, et Buffon (par prudence peut-être) s'en tient à une image de la nature où les espèces coexistent de toute éternité les unes à côté des autres. Les gradations, les nuances qu'il aperçoit partout ne découlent donc pas les unes des autres selon un ordre causal et successif; elles sont toutes simultanées, et l'immense collectivité du monde vivant n'est pas entraînée par le devenir. De surcroît, entre le plus perfectionné des animaux et l'homme, il voit s'interrompre la continuité graduelle et insensible qui unissait toutes les espèces: on ne passe de l'animal à l'homme que par un saut qualitatif brusque. Qu'il y ait là une satisfaction donnée aux autorités religieuses, c'est probable, et il n'est pas impossible que Buffon ait voulu se protéger contre le renouvellement des attaques qu'avait provoquées sa Théorie de la Terre. Rousseau, plus sincèrement sans doute, affirme aussi cette frontière métaphysique: l'animal n'est «qu'une machine ingénieuse », ${ }^{12}$ tandis que l'homme est doué de liberté. Seulement Rousseau situera cette frontière plus bas dans l'échelle des êtres connus. En adversaire du matérialisme, il reconnaît une radicale différence d'essence entre le singe et l'homme, par quoi il s'oppose aux idées hardies de La MetTrie; toutefois la question se pose pour lui de savoir si l'ourang-outang et le mandrille sont effectivement des singes. Dans le doute, il préfère trancher par la négative: ce ne sont peut-être pas des animaux, mais des hommes tout à fait primitifs, comparables aux «satyres» des anciens et aux «hommes

12 Rousseau, Euvres complètes, édition Hachette, t. I, p. 89. 
sylvestres» des savants de la Renaissance. Rousseau n'est pas le seul en son temps à élargir ainsi les limites de l'humanité. LinNé, lui aussi, rangeait dans le genre humain certains anthropoïdes, auxquels il donnait le nom spécifique d'homo nocturnus.

Accueillant dans le genre humain des créatures si différentes de l'homme civilisé, Rousseau fait intervenir un écart considérable entre l'homme primitif et l'Européen policé. Cet écart, il faut l'expliquer par une histoire qui altère et transforme sinon la nature même de l'homme, du moins sa «constitution». L'homme devient alors un exemple particulièrement éloquent du transformisme restreint dont Buffon avait si bien décrit les étapes pour les espèces animales modifiées par l'art humain et qu'il n'avait pas hésité à attribuer aussi à l'espèce humaine. Les phrases initiales de l'Emile établissent une complète analogie entre les transformations que l'homme s'impose à lui-même et celles qu'il fait subir à des espèces naturelles telles que le chien et le cheval. Buffon, dans les Epoques de la Nature, insistera à son tour sur la simultanéité des actions que l'homme exerce sur la nature et sur lui-même; il y verra un développement heureux; la connaissance rationnelle, les techniques qui en découlent, éduquent et corrigent la nature pour le bien de l'humanité, permettant ainsi à l'homme de se perfectionner. Nous sommes loin du regret de Rousseau: «Tout dégénère entre les mains de l'homme.» L'histoire n'apparaît pas à Buffon comme un risque mortel, mais comme la succession des étapes à travers lesquelles l'homme prend possession de la plénitude de ses pouvoirs en disposant souverainement de toutes les richesses du monde naturel. La civilisation, pour Buffon, est l'épanouissement normal de l'humanité de l'homme. L'entendement, l'intelligence, la société ne sont pas, selon lui, des acquisitions historiques, mais des propriétés essentielles de l'homme: elles appartiennent déjà au sauvage. Il n'y aura donc pas, pour Buffon, d'opposition frappante entre l'état de nature et l'état de civilisation. Chez Rousseau, en revanche, l'écart entre ces deux états est presque aussi considérable qu'entre l'homme et l'animal. C'est pourquoi il s'autorise à modifier l'analyse différentielle par laquelle Buffon réservait au sens spirituel tout ce qui ne pouvait être expliqué par les opérations du «sens intérieur matériel». La méthode de Rousseau dans le second Discours consistera à définir exhaustivement l'homme de la nature, au physique et au moral, de façon à rendre plus nettement évidentes les acquisitions historiques, les adjonctions factices, les pouvoirs surnuméraires qui sont les produits de notre propre activité. La méthode de Rousseau est celle de Buffon, sauf que la confrontation principale s'établit entre l'homme 
d'aujourd'hui et l'homme d'un passé immémorial (entre deux moments extrêmes de l'évolution humaine) et non plus entre l'entendement et l'animalité. «Il est nécessaire d'avoir des notions justes» de l'état originaire de l'homme, "pour bien juger de notre état présent. ${ }^{13} \mathrm{~L}$ 'une des différences majeures apparaît ici: Buffon s'efforce de distinguer, hors de toute considération temporelle, les opérations qui appartiennent à la sphère du corps et celles qui sont $d u$ ressort de la raison. Rousseau, pour sa part, tenant pour acquise la distinction de l'homme et de l'animal, du corps et de l'âme, ne fait plus intervenir un écart métaphysique, mais un écart historique entre les termes qu'il confronte: il oppose à l'homme moderne l'image d'un homme qui appartient au passé le plus profond. Ainsi s'introduit une tension historique que Buffon ne pressentait pas.

Toute la première partie du Discours de l'Inégalité s'applique à dépeindre un être qui n'a encore exercé ses facultés ni pour vaincre les obstacles extérieurs ni pour se transformer lui-même. Ceci constaté, on saura mieux reconnaître, en nous, ce qui appartient à la nature et ce qui est «l'homme de l'homme». Entre ce qu'était l'homme de la nature et ce que nous sommes devenus, un drame s'est joué, dont la conséquence se perpétue dans nos conflits intérieurs.

Il vaut la peine de le remarquer: Rousseau, reprenant la méthode de Buffon pour s'en servir à sa guise, n'oublie rien de ce que Buffon a dit de la condition animale. Seulement Rousseau reporte sur l'homme de la nature le bonheur physique que Buffon attribue à l'animal. Il n'y a là, à première vue, aucune contradiction. Selon Buffon, tout ce que possède la bête, l'homme le possède aussi ; et, dans la première enfance ou dans l'état d'imbécilité, il ne possède guère que les facultés animales. L'homme sauvage, selon Rousseau, goûte la plénitude sans exercer les pouvoirs de l'entendement, dont Buffon faisait le caractère spécifique et obligatoire de l'âme humaine. Le sauvage de Rousseau sera complètement homme en l'absence de toute activité intellectuelle ou technique. Le seul caractère qui le distingue de l'animal est sa liberté: encore reste-t-elle sans emploi, puisque l'homme n'est engagé dans aucune activité pratique. L'on pourra donc dire que Rousseau, pour peindre l'homme de la nature, animalise et «désintellectualise» l'homme qu'avait décrit Buffon; l'on constatera en revanche qu'il humanise et spiritualise un certain nombre de sentiments que Buffon reléguait dans le domaine obscur du «sens intérieur matériel».

${ }^{13}$ Rousseau, Euvres complètes, édition Hachette, t. I, p. 79. 
Comment l'homme, selon Buffon, prend-t-il conscience de son existence? L'homme se sent exister d'autant plus qu'il ajoute, par la mémoire, le souvenir de son existence passée au sentiment de son existence actuelle: «La conscience de son existence, ce sentiment intérieur qui constitue le moi, est composé chez nous de la sensation de notre existence actuelle et du souvenir de notre existence passée. Ce souvenir est une sensation tout aussi présente que la première, elle nous occupe même quelquefois plus fortement et nous affecte plus puissamment que les sensations actuelles; et comme ces deux espèces de sensations sont différentes et que notre âme a la faculté de les comparer et d'en former des idées, notre conscience d'existence est d'autant plus certaine et d'autant plus étendue, que nous nous représentons plus souvent et en plus grand nombre les choses passées... Il est évident que plus on a d'idées, plus on est sûr de son existence; que plus on a d'esprit, plus on existe; qu'enfin c'est par la puissance de réfléchir qu'a notre âme, et par cette seule puissance, que nous sommes certains de nos existences passées et que nous voyons nos existences futures. ${ }^{14}$

Au contraire de ce qu'affirme Buffon, l'homme de la nature (et JeanJacques lui-même) n'ont pas besoin d'idées pour sentir fortement leur existence; au contraire, ils la sentent d'autant mieux qu'ils ignorent ou qu'ils font taire l'activité de la réflexion: «Son âme, que rien n'agite, se livre au seul sentiment de son existence actuelle, sans idée de l'avenir, quelque prochain qu'il puisse être. ${ }^{15}$ L'existence actuelle, la seule existence momentanée, c'est aussi ce que l'animal perçoit de lui-même, et c'est du même coup aux yeux de Buffon, ce qui rend cette forme de conscience de soi imparfaite et bornée, en regard de la connaissance plus complète que l'homme prend de son existence: "[Les animaux] ont... la conscience de leur existence actuelle, mais ils n'ont pas celle de leur existence passée... La puissance de réfléchir ayant été refusée aux animaux, il est donc certain qu'ils ne peuvent former d'idées et que par conséquent leur conscience est moins sûre et moins étendue que la nôtre; car ils ne peuvent avoir aucune idée du temps, aucune connaissance du passé. aucune notion de l'avenir; leur conscience d'existence est simple; elle dépend uniquement des sensations qui les affectent actuellement et consiste dans le sentiment intérieur que ces sensations produisent... Ils ne savent point qu'ils existent, mais ils le

${ }^{14}$ Buffon, t. II, p. $336 \mathrm{f}$.

${ }^{15}$ Rousseau, CEuvres complètes, édition Hachette, t. I, p. 91. 
sentent. ${ }^{16}$ Or tout se passe, pour Rousseau, comme si la privation du savoir et de la prévoyance était un véritable privilège.

Par une singulière conséquence, Buffon compare «cette conscience d'existence dans les animaux» à "l'état où nous nous trouvons lorsque nous sommes fortement occupés d'un objet, ou violemment agités par une passion qui ne nous permet de faire aucune réflexion sur nous-mêmes. On exprime l'idée de cet état en disant qu'on est hors de soi, et l'on est en effet hors de soi dès que l'on n'est occupé que des sensations actuelles ${ }^{17}$. La véritable intériorité, ou du moins la possession de soi, est liée pour Buffon à l'exercice de l'entendement, à la mémoire active et volontaire qui nous rattache au passé; elle implique également la prévision du futur. Pour Rousseau, bien au contraire, c'est le retour actif sur le passé et c'est surtout le souci de l'avenir, la prévoyance, qui nous arrachent à nous-mêmes et nous font vivre hors de nous: la réflexion, qui compare les objets, qui confronte les moments divers de notre expérience, nous invite aussi à nous opposer aux autres et à nous chercher dans les regards des autres. Elle nous aliène. Par conséquent, Rousseau affirme: «Le sauvage vit en lui-même; l'homme sociable, toujours hors de lui, ne sait vivre que dans l'opinion des autres, et c'est, pour ainsi dire, de leur seul jugement qu'il tire le sentiment de sa propre existence. ${ }^{18} \mathrm{Si}$ vivre dans le sentiment de l'existence actuelle équivaut à vivre en soi-même, l'homme de la nature selon Rousseau réalise spontanément un idéal d'indépendance que l'homme civilisé ne peut atteindre qu'au terme d'un long effort philosophique: « [L'homme de la nature] ne veut que vivre et rester oisif, et l'ataraxie même du Stoïcien n'approche pas de sa profonde indifférence pour tout autre objet. ${ }^{19}$ Les effets néfastes de la passion, dès lors, n'apparaissent plus comme la turbulence de notre animalité (thèse soutenue par Buffon), mais comme les conséquences d'une réflexion malheureuse qui convoite des satisfactions sans nul rapport avec les besoins naturels de l'individu. Rousseau, peut de la sorte, reprendre à son compte les fameuses considérations de Buffon, sur l'amour: «Il n'y a que le physique de cette passion qui soit bon; ...malgré ce qu'en peuvent dire les gens épris, le moral n'en vaut rien. Qu'est-ce en effet que le moral de l'amour? la vanité... ${ }^{20}$ Rousseau reportera donc sur l'homme de la

${ }^{16}$ Buffon, t. II, p. 336-8.

${ }^{17}$ Buffon, t. II, p. 338.

${ }^{18}$ Rousseau, Euvres complètes, édition Hachette, t. I, p. 126.

${ }^{19}$ Rousseau, Euvres complètes, édition Hachette, t. I, p. 125

${ }^{20}$ Buffon, t. II, p. 352. 
nature l'éloge lyrique que Buffon adressait à l'équilibre heureux du désir animal: «Les animaux ne sont point sujets à toutes ces misères; ils ne cherchent pas des plaisirs où il ne peut y en avoir: guidés par le sentiment seul, ils ne se trompent jamais dans leur choix; leurs désirs sont toujours proportionnés à la puissance de jouir, ils sentent autant qu'ils jouissent, et ne jouissent qu'autant qu'ils sentent. L'homme, au contraire, en voulant inventer des plaisirs, n'a fait que gâter la nature; en voulant se forcer sur le sentiment, il ne fait qu'abuser de son être, et creuser dans son cœur un vide que rien ensuite n'est capable de remplir. ${ }^{21}$

Constatons que le système de Rousseau, en l'occurrence, s'ḩarmonise mieux avec cette page de Buffon que ne le fait, chez Buffon, le dualisme de façade. Car n'y a-t-il pas une contradiction à affirmer que seul le physique de l'amour est bon et, à quelques pages de distance, que le principe spirituel en nous est une lumière pure accompagnée par «le calme et la sérénité», tandis que le principe matériel « est une fausse lueur qui ne brille que par la tempête et dans l'obscurité, un torrent impétueux qui roule et entraîne à sa suite les passions et les erreurs " ? ${ }^{22}$ Rousseau, faisant de la réflexion une puissance ambiguë qui perfectionne l'homme en l'aliénant, peut laisser à l'animalité de l'homme toute son innocence; il attribuera la responsabilité du mal à «l'entendement en délire ». ${ }^{23}$ Les descriptions du malheur de l'homme policé que nous trouvons dans le Discours sur la Nature des Animaux ne s'accordent guère avec l'orgueil satisfait que Buffon, dans le reste de son œuvre, manifeste toutes les fois qu'il évoque la domination de l'homme sur la nature; elles illustrent mieux le pessimisme historique de Rousseau que l'optimisme rationaliste de Buffon. Il est plus cohérent, en effet, d'attribuer le malheur de l'homme aux qualités spécifiques de l'humanité que d'incriminer, sans en analyser l'origine, «le dérèglement de notre sens intérieur matériel». ${ }^{24}$ En déplaçant seulement la cause du mal, Rousseau peut tout reprendre des pages véhémentes de Buffon: «Dans l'homme, le plaisir et la douleur physique ne sont que la moindre partie de ses peines et de ses plaisirs: son imagination, qui travaille continuellement, fait tout, ou plutôt ne fait rien que pour son malheur; car elle ne présente à l'âme que des fantômes vains ou des images exagérées... Nous nous préparons donc des peines toutes les fois que nous cherchons des plaisirs. Nous sommes

21 Ibid.

22 Buffon, t. II, p. 346.

23 Rousseau, Euvres complètes, édition Hachette, t. I, p. 79.

${ }^{24}$ Buffon, t. II, p. 335. 
malheureux dès que nous désirons d'être plus heureux... Il y a dans le physique infiniment plus de bien que de mal: ce n'est pas la réalité, c'est la chimère qu'il nous faut craindre; ce n'est ni la douleur du corps, ni les maladies, ni la mort, mais l'agitation de l'âme, les passions et l'ennui qui sont à redouter... Nous cherchons à nous détruire en cherchant à forcer la nature, nous ne savons pas trop ce qui nous convient ou ce qui nous est nuisible; nous ne distinguons pas bien les effets de telle ou telle nourriture; nous dédaignons les aliments simples, et nous leur préférons des mets composés, parce que nous avons corrompu notre goût, et que d'un sens de plaisir nous avons fait un organe de débauche qui n'est flatté que de ce qui l'irrite.» ${ }^{25}$

Ce que Jean-Jacques ne répétera pas, c'est l'explication donnée par Buffon: «Notre âme ne nous a été donnée que pour connaître, nous ne voudrions l'employer qu'à sentir. ${ }^{26}$ S'il y a dans le physique, comme l'a dit Buffon, infiniment plus de bien que de mal, comment le mal viendrait-il de notre désir de sentir, c'est-à-dire de nous confiner dans le physique? Le projet d'une morale sensitive prouve bien que pour Rousseau la vie heureuse se conçoit comme une immersion ou une réintégration dans un univers sensible, préalablement agencé et aménagé par une réflexion raisonnable qui aura su reconnaître l'empire du sensible. Buffon pose une contradiction que Rousseau tend à résoudre dialectiquement en faisant de la réflexion, d'abord, le trouble-fête et l'instigatrice du mal, mais à un stade ultérieur, quand l'entendement devient raison éclairée, un pouvoir de réconciliation.

L'idée de l'unité qui importe tant à Rousseau se trouve pourtant nettement affirmée dans Buffon, qui écrit: «L'homme sage est sans doute l'être le plus heureux de la nature, il joint aux plaisirs du corps, qui lui sont communs avec les animaux, les joies de l'esprit, qui n'appartiennent qu'à lui: il a deux moyens d'être heureux qui s'aident et se fortifient mutuellement. ${ }^{27}$ Rousseau lui aussi voit l'homme divisé, mais ne se contente pas de proclamer la nécessité d'une hégémonie de la raison: la sagesse, c'est-à-dire l'unité retrouvée, n'est réalisable qu'au terme d'un long effort, où la raison se conquiert et se transforme en s'efforçant de conserver intacte (ou de sauver du moins par le souvenir) l'image d'une nature et d'une quiétude perdues, qui seront restaurées sur le plan supérieur de la vie morale et de la vie sociale.

${ }^{25}$ Buffon, t. II, p. 332-5.

${ }^{26}$ Buffon, t. II, p. 333.

${ }^{27}$ Buffon, t. II, p. 334. 
Buffon a répondu à Rousseau avant d'avoir connu l'Emile. Dans le préambule qui précède l'Histoire Naturelle des Carnassiers (1758), il réfutera les hypothèses de Rousseau sur l'homme de la nature. En quoi il ne sera que fidèle à lui-même: dans le Discours sur la Nature des Animaux, il avait déjà affirmé que «l'homme n'est homme que parce qu'il a su se réunir à l'homme », et «que tout a concouru à rendre l'homme sociable». ${ }^{28} \mathrm{Il}$ y avait ajouté les arguments traditionnels sur la nécessité d'une société rudimentaire (la famille) pour que le nouveau-né survive jusqu'au moment de l'indépendance. Or Rousseau s'était appliqué à réfuter ces arguments, empruntant parfois à Buffon des observations favorables à l'hypothèse d'une dispersion complète des individus primitifs. Répliquant dans le préambule des Animaux Carnassiers, Buffon refuse à nouveau le postulat de la solitude de l'homme naturel, et, d'autre part, jette le doute sur la vision évolutive de l'histoire humaine: «Nous avons sous les yeux, non l'état idéal, mais l'état réel de la nature. Le sauvage habitant des déserts est-il un animal tranquille? est-il un homme heureux? car nous ne supposerons pas avec un philosophe, l'un des plus fiers censeurs de notre humanité, qu'il y a une plus grande distance de l'homme en pure nature au sauvage, que du sauvage à nous; que les âges qui se sont écoulés avant l'invention de l'art de la parole ont été bien plus longs que les siècles qu'il a fallu pour perfectionner les signes et les langues, parce qu'il me paraît que, lorsqu'on veut raisonner sur des faits, il faut éloigner les suppositions et se faire une loi de n'y remonter qu'après avoir épuisé tout ce que la nature nous offre... ${ }^{29}$ »

Buffon se rabat prudemment sur les faits aujourd'hui constatables. Alors qu'il n'avait pas hésité à remonter par hypothèse dans le passé physique du globe terrestre, il répugne à hasarder la même démarche conjecturale en ce qui concerne le début de l'espèce humaine. La résistance d'un Buffon, mieux que tout autre document, donne parfaitement la mesure de l'audace et de la nouveauté du Discours de l'Inégalité.

${ }^{28}$ Buffon, t. II, p. 359.

${ }^{29}$ Buffon, t. II, p. 556 sq. 